\title{
Oxidation Decomposition of Unsaturated Fatty Acids by Singlet Oxygen in Phospholipid Bilayer Membranes
}

\author{
Naoki Watabe ${ }^{1, \$}$, Yu Ishida ${ }^{1, \#}$, Akira Ochiai ${ }^{1}$, \\ Yoshikazu Tokuoka ${ }^{1 *}$ and Norimichi Kawashima ${ }^{1,2}$ \\ ${ }^{1}$ Faculty of Biomedical Engineering, Toin University of Yokohama (1614 Kurogane-cho, Aoba-ku, Yokohama 225-8502, JAPAN) \\ ${ }^{2}$ Biomedical Engineering Center, Toin University of Yokohama (1614 Kurogane-cho, Aoba-ku, Yokohama 225-8502, JAPAN)
}

\begin{abstract}
Oxidation decomposition of unsaturated fatty acids with singlet oxygen generated from a photosensitizing agent was investigated in liposome bilayer membranes under a light irradiation condition. The liposome of which the bilayer membrane was composed of $L$ - $\alpha$-dipalmitoylphosphatidylcholine (DPPC), protoporphyrin IX (PpIX), and an unsaturated fatty acid (oleic acid, linoleic acid, $\alpha$-linolenic acid, or arachidonic acid) were prepared with Bangham's method. In irradiating the liposome dispersion with light ranged from 550 to $750 \mathrm{~nm}$, the unsaturated fatty acid was decomposed through an oxidation reaction with singlet oxygen. The decomposition rate constant was obeyed as the following order: arachidonic acid $>$ oleic acid $>\alpha$-linolenic acid $>$ linoleic acid. This result indicates that oleic acid is readily degraded despite its lower unsaturated degree. In addition, micropolarity and microfluidity of the hydrocarbon region in the liposome bilayer membrane including the unsaturated fatty acid and PpIX decreased with an increase in light irradiation time. These findings suggest that interaction among the hydrocarbon chains of DPPC in the liposome bilayer membrane is promoted by migration of the oxidized unsaturated fatty acid from the hydrocarbon region, leading to form close-packed and well-ordered orientation of the hydrocarbon chains.
\end{abstract}

Key words: oxidation decomposition, unsaturated fatty acid, singlet oxygen, phospholipid bilayer membrane, protoporphyrin IX

\section{INTRODUCTION}

Photodynamic therapy (PDT), which is one of the most useful cancer therapies, includes administration and localization of photosensitizing agents, porphyrins, chlorines, etc., into cancer tissues, followed by activation of the agent by light with a specific wavelength. The photoexited photosensitizing agent transfers the energy to molecular triplet oxygen to form singlet oxygen ${ }^{1)}$, thus leading to photochemical and photobiological processes that cause irreversible photodamage, apoptosis and necrosis, to the cancer tissues ${ }^{2,3)}$.

Phospholipids are one of main compositions of biological membranes. The phospholipid has two asymmetric hydrocarbon chains; one is saturated and the other is mono-cisor poly-cis-unsaturated. Since the unsaturated hydrocarbon chain has a pronounced bend at a cis-double bond, it prevents close-packed and well-ordered orientation of the hydrocarbon chains of the phospholipids, which raises fluidity of the biological membrane ${ }^{4)}$.

Singlet oxygen readily oxidizes a cis-double bond of unsaturated hydrocarbon chains and introduces hydroperoxy group into them. In addition, some porphyrins employed as a photosensitizing agent in PDT temporarily accumulate in mitochondrial and cell membranes ${ }^{5}$. Singlet

\footnotetext{
* Correspondence to: Yoshikazu Tokuoka, Faculty of Biomedical Engineering, Toin University of Yokohama, 1614 Kurogane-cho, Aoba-ku, Yokohama 225-8502, JAPAN

E-mail: tokuoka@cc.toin.ac.jp

${ }^{\$}$ Current address: Human R\&D Co., Ltd., Yokohama-Kanazawa High-Tech Center Bldg., 6F, Fukuura 1-1-1, Kanazawa-ku, Yokohama 2360004, JAPAN

"Current address: Fukuda Denshi, Co., Ltd., Hongo 2-35-8, Bunkyou-ku, Tokyo 113-8420, JAPAN

Accepted September 23, 2006 (received for review July 24, 2006)
}

Journal of Oleo Science ISSN 1345-8957 print / ISSN 1347-3352 online

http://jos.jstage.jst.go.jp/en/ 
oxygen generated from the photoexited porphyrin then reacts to a cis-double bond of phospholipids in the membrane. Therefore, it is quite important in PDT to investigate oxidization of unsaturated hydrocarbon chains by singlet oxygen in phospholipid bilayer membranes and to realize influence of the oxidized unsaturated hydrocarbon chain on membrane properties.

It is well known that oxidation reactions of unsaturated fatty acids with active oxygen species are accelerated with an increase in their unsaturated degrees in homogeneous solutions. On the contrary, Yazu et al. have observed that oxidizability of unsaturated fatty acids solubilized in aqueous surfactant micellar phases by active oxygen species decreases with an increase in the number of their double bonds ${ }^{6,7}$. Moreover, Kobayashi et al. have reported that unsaturated fatty acids with higher unsaturated degrees are little susceptible to their oxidation by active oxygen species in micellar phases ${ }^{8}$. In these studies, they used water-soluble azo compounds as an oxidation initiator and focused on the oxidizability of the unsaturated fatty acid in the surfactant micellar phase by the active oxygen species coming from the water bulk phase.

In the present study, we prepared liposomes of which bilayer membranes contained phospholipids, a photosensitizing agent, and an unsaturated fatty acid with various unsaturated degrees, and determined an oxidation decomposition rate of the unsaturated fatty acid by singlet oxygen under a light irradiation condition. We then discuss the relationship between the oxidation decomposition rate and the unsaturated degree of the unsaturated fatty acid in the phospholipid bilayer membrane. Further, physicochemical properties of the phospholipid bilayer membrane after the oxidation reaction were investigated.

\section{EXPERIMENTAL PROCEDURE}

\subsection{Materials}

L- $\alpha$-dipalmitoylphosphatidylcholine (DPPC, 99.7\%) was purchased from NOF. Co. Protoporphyrin IX (PpIX), which was purchased from Aldrich Co., was employed as a photosensitizing agent. They were used without further purification. Pyrene (98\%) as a fluorescence probe was purchased from WAKO Pure Chemical Industries, Ltd., and was used after triple extraction with ethanol. Unsaturated fatty acids, oleic acid, linoleic acid, $\alpha$-linolenic acid, and arachidonic acid, were purchased from Aldrich Co., and were used without further purification.

\subsection{Preparation of liposome with phospholipid bilayer membrane including unsaturated fatty acid and PpIX}

Liposomes of which bilayer membranes contained DPPC, PpIX, and an unsaturated fatty acid were prepared using Bangham's method ${ }^{9}$. These compounds were dissolved in 5
$\mathrm{mL}$ of chloroform in a test tube to obtain a given concentration: $2.5 \times 10^{-5} \mathrm{M}$ of DPPC, $1.0 \times 10^{-5} \mathrm{M}$ of the unsaturated fatty acid, and $1.0 \times 10^{-6} \mathrm{M}$ of PpIX. Chloroform was evaporated under $\mathrm{N}_{2}$ gas, and a thin lipid film was formed on the wall of the test tube. In order to remove a residual solvent completely, the test tube was allowed to stand in a desiccator under reduced pressure. After $12 \mathrm{~mL}$ of distilled water was added into the test tube, the test tube was kept for $5 \mathrm{~min}$ at $60^{\circ} \mathrm{C}$, agitated on a vortex mixer for a few minutes to give liposome dispersion. The liposome dispersion was extruded through a $10 \mu \mathrm{m}$ of Millipore filter (Whatman Co.) with an extruder (Lipex Biomembranes Inc.) to obtain homogeneously sized liposome dispersion. The liposome formation was confirmed with light and fluorescence microscopy.

\subsection{Determination of singlet oxygen generated in liposome bilayer membrane}

A $1.0 \mathrm{~mL}$ of $0.3 \mathrm{M}$ 2,2,6,6-tetramethyl-4-piperidone hydrochloride (TMPD, Kanto Chemical Co., Ltd.) solution was added into $1.0 \mathrm{~mL}$ of homogenously sized liposome dispersion that had been prepared by using DPPC and PpIX, but without an unsaturated fatty acid. The mixture was exposed to light emitted from a metal-halide lamp for a given time at $25^{\circ} \mathrm{C}$. The wavelength of the light ranged from 550 to $750 \mathrm{~nm}$. Under this irradiation condition, the liposomal structure was maintained. ESR spectra of the mixture were measured with an ESR spectrometer (JESAF200, JEOL) before and after the light irradiation. ESR spectra of the liposome dispersion with histidine, which can scavenge singlet oxygen, were also measured. In general, TMPD is oxidized by singlet oxygen and is converted to a stable nitroxide radical $(>\mathrm{N}-\mathrm{O} \cdot)$ that gives a characteristic ESR signal ${ }^{10}$, but the ESR signal of the nitroxide radical disappears by adding histidine ${ }^{11)}$.

\subsection{Decomposition of unsaturated fatty acid by singlet oxy- gen in liposome bilayer membrane}

After exposing homogeneously sized liposome dispersion including PpIX and an unsaturated fatty acid to light emitted from the metal-halide lamp for a given time at $25^{\circ} \mathrm{C}$, we confirmed with microscopy that the liposomal structure was maintained in the dispersion. Afterward, we broke the liposome by adding ethanol. A concentration of a remaining unsaturated fatty acid was determined with a high performance liquid chromatograph (HPLC, LaChrom, HITACH, Ltd.) with an octadecylsilane column $(250 \mathrm{~mm}$ in length, $4.6 \mathrm{~mm}$ in diameter) and an ethanol-water mixed solution (volume ratio of ethanol to water: 4:1) as eluent.

\subsection{Measurement of micropolarity and microfluidity of lipo- some bilayer membrane using fluorescence probe method with pyrene}

Homogeneously sized liposome dispersion with $6.0 \times 10^{-3}$ 
M of pyrene was prepared by the same method as described before. Before and after light irradiation, fluorescence spectra of pyrene in the liposome bilayer membrane were measured with a fluorescence spectrophotometer (V530, JASCO Co.) at $25^{\circ} \mathrm{C}$. The excitation wavelength was $335 \mathrm{~nm}$, and the emission wavelength was in the range from 350 to $650 \mathrm{~nm}$. The fluorescence peak intensity ratio of pyrene at 375 and $395 \mathrm{~nm}\left(\mathrm{I}_{1} / \mathrm{I}_{3}\right)$ reflects micropolarity around pyrene ${ }^{12,13)}$; the micropolarity increases with an increase in $\mathrm{I}_{1} / \mathrm{I}_{3}$ of pyrene. In addition, the face-to-face collision of pyrene monomers often forms pyrene excimers above a certain concentration of pyrene ${ }^{12)}$. The pyrene fluorescence peaks at 395 and $473 \mathrm{~nm}$ are assigned to pyrene monomers and excimers, respectively. The fluorescence peak intensity ratio between excimers and monomers $\left(\mathrm{I}_{\mathrm{E}} / \mathrm{I}_{\mathrm{M}}\right)$ indicates microfluidity around pyrene ${ }^{14)}$; the larger the $I_{E} / I_{M}$ of pyrene is, the higher the microfluidity around pyrene is.

\section{RESULTS AND DISCUSSION}

3.1 Generation of singlet oxygen in liposome bilayer membrane

We prepared liposomes using DPPC and PpIX, but without an unsaturated fatty acid, and determined generation of singlet oxygen in the liposome dispersion under a light irradiation condition. Fig. 1 (a) and (b) show ESR spectra of the liposome dispersion with TMPD before and after the

(a)

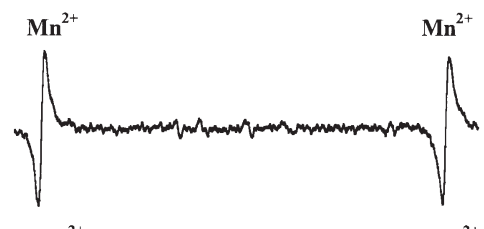

(b)

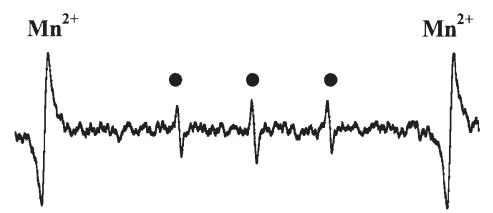

(c)

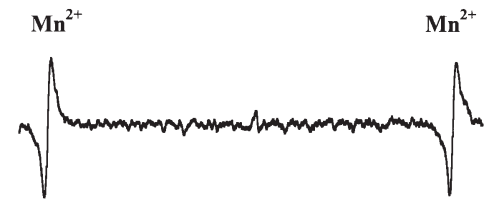

Fig. 1 ESR Spectra of Liposome Dispersion with TMPD before and after Light Irradiation at $25^{\circ} \mathrm{C}$ : (a) and (b) Non-Light-Irradiated and Light-Irradiated Liposome Dispersion, Respectively, (c) HistidineContaining Liposome Dispersion Irradiated with Light. Closed Circles Shows ESR Signal of TMPD Nitroxide Radical. light irradiation for 5 min, respectively. Besides, Fig. 1 (c) depicts an ESR spectrum of the liposome-TMPD mixed dispersion with histidine after the 5-min light irradiation. We detected no ESR signals before the light irradiation, but a characteristic ESR signal with three equal intensity lines (closed circles in Fig. 1 (b)) assigned to a TMPD nitroxide radical after the irradiation. When histidine was added, the ESR signal of the TMPD nitroxide radical disappeared despite the light irradiation. When exposing the water bulk phase with TMPD to the light, we detected no ESR signals (the data is not shown). In addition, fluorescence microscopy for the liposome dispersion observed the PpIX fluorescence from the liposome bilayer membrane, but not from the water bulk phase. Taking account of these findings, we consider that PpIX exists in the liposome bilayer membrane and that singlet oxygen is generated from PpIX in the membrane, but not in the water bulk phase.

Fig. 2 expresses the relative intensity of the ESR signal of the TMPD nitroxide radical against that of the manganese markers on the both sides of the ESR spectra shown in Fig. 1. The relative intensity corresponds to a quantity of singlet oxygen generated ${ }^{15}$. The relative intensity shown in Fig. 2 rises with irradiation time and becomes almost constant over 10-min light irradiation. As a result, singlet oxygen generation is restricted in light irradiation above $10 \mathrm{~min}$.

Photosensitizers including PpIX are generally bleached in light irradiation. They are decomposed into their oxidants ${ }^{16)}$. In order to understand the bleaching of PpIX in the liposome bilayer membrane under this experimental condition, we measured fluorescence spectra of PpIX after

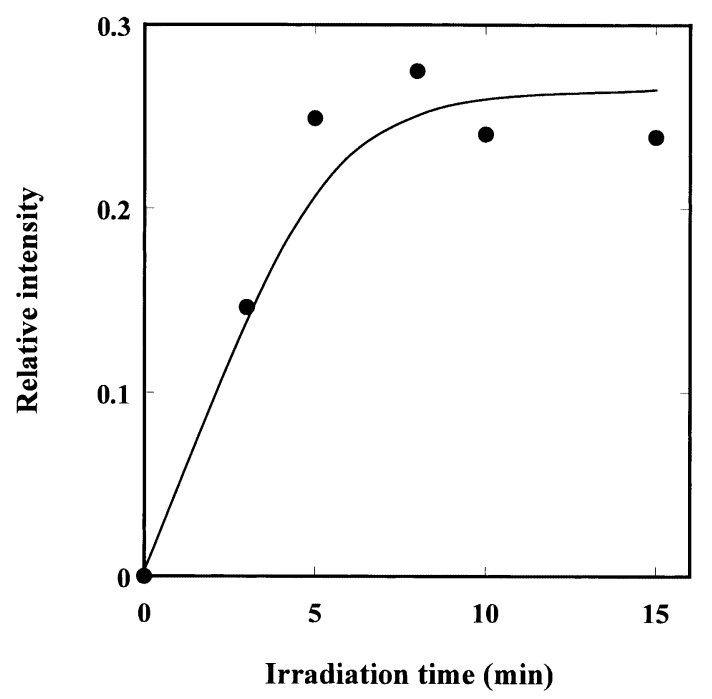

Fig. 2 Change in Relative Intensity of ESR Signal of TMPD Nitroxide Radical to that of Manganese with Light Irradiation Time at $25^{\circ} \mathrm{C}$. 
light irradiation. Fig. 3 depicts irradiation time-dependence of fluorescence peak intensity at $633 \mathrm{~nm}$ of PpIX in liposome dispersion excited by light at $410 \mathrm{~nm}$. Here, the vertical line corresponds to the fluorescence intensity ratio of PpIX with and without the light irradiation, namely, a remaining PpIX concentration ratio in the liposome dispersion. As can be seen from Fig. 3, the concentration of PpIX in the membrane is gradually reduced with increasing light irradiation time. It is probably that this reduction induces the restriction of the singlet oxygen generation shown in Fig. 2.

\subsection{Decomposition of unsaturated fatty acid by singlet oxy- gen in liposome bilayer membrane}

Fig. 4 refers to changes in concentration of the unsaturated fatty acid in the liposome bilayer membrane with light irradiation time. Here, the vertical line corresponds to the ratio of the unsaturated fatty acid concentration at a given irradiation time against that at the initial state (irradiation time: 0). The concentration ratio decreases with increasing irradiation time in any system. We also confirmed no decomposition of the unsaturated fatty acid without light irradiation or PpIX (the data is not shown). These findings then consider that the decomposition of the unsaturated fatty acid is caused by an oxidation reaction with singlet oxygen generated through PpIX in the phospholipid bilayer membrane.

We estimate the correlation between the decomposition rate and the unsaturated degree of the unsaturated fatty acid by using oxidation kinetics. Oxidation decomposition of unsaturated fatty acids by singlet oxygen is essentially conformed to a second-order reaction for a concentration of the unsaturated fatty acid and singlet oxygen. Not

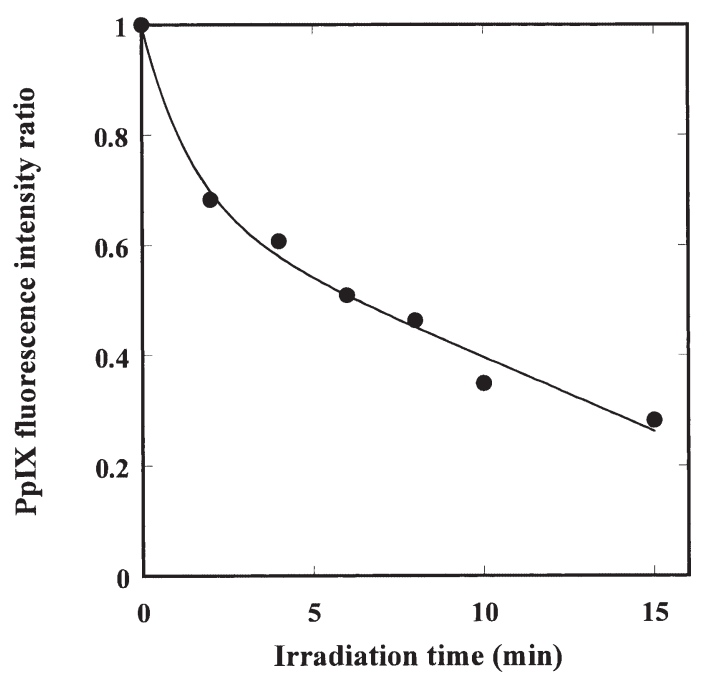

Fig. 3 Change in PpIX Fluorescence Intensity Ratio with and without Light Irradiation as a Function of Light Irradiation Time at $25^{\circ} \mathrm{C}$. detecting concentrations of singlet oxygen during the oxidation reaction in our experiment, however, we regard the oxidation reaction as an apparent first-order reaction for the unsaturated fatty acid concentration ratio shown in Fig. 4. We summarize the results obtained from the apparent first-order reaction in Fig. 5. There is a nonlinear rela-

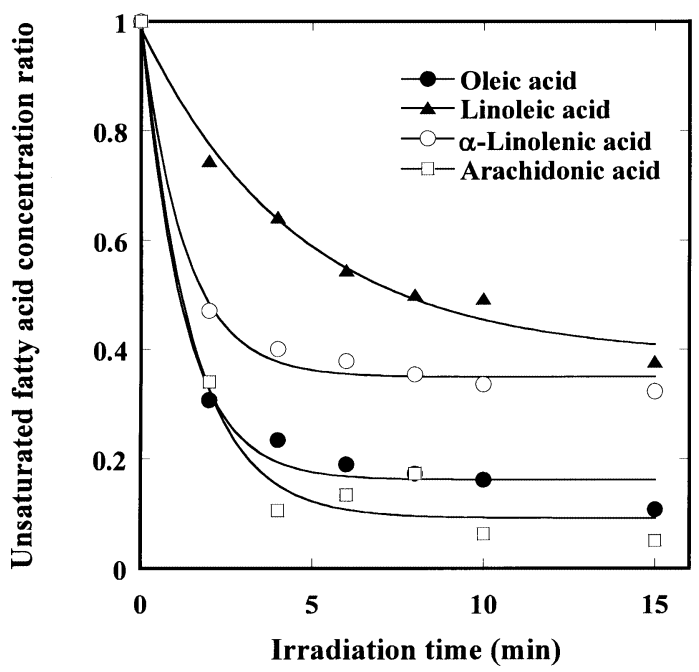

Fig. 4 Changes in Concentration Ratio of Unsaturated Fatty Acid with and without Light Irradiation as a Function of Light Irradiation Time at $25^{\circ} \mathrm{C}$.

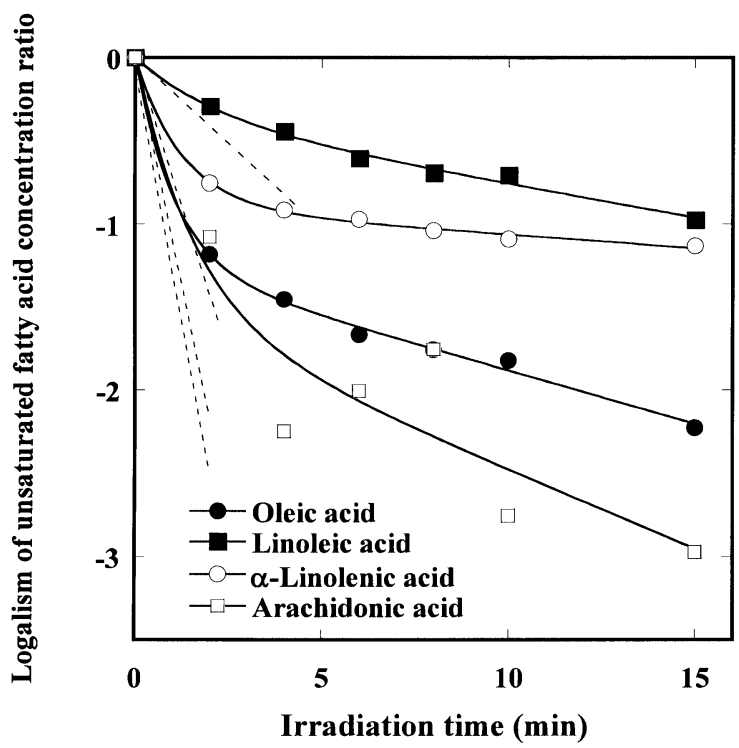

Fig. 5 Relationship between Unsaturated Fatty Acid Concentration Ratio in Liposome Bilayer Membrane and Light Irradiation Time Evaluated by Apparent First-Order Reaction for Unsaturated Fatty Acid Concentration Ratio at $25^{\circ} \mathrm{C}$. Dashed lines demonstrate slopes of the relationship at the initial state (irradiation time: 0 ). 
tionship in any system, and the slope, or the decomposition rate constant, gradually becomes smaller with increasing irradiation time. This is probably caused by the fact that it gets hard to generate singlet oxygen with increasing irradiation time demonstrated in Fig. 2

Fig. 6 refers to change in decomposition rate constant of the unsaturated fatty acid at the initial state (irradiation time: 0 ) as a function of its unsaturated degree. The decomposition rate constant was estimated from the slope at the initial state, drawn by a dashed line in Fig. 5, on the assumption that the same quantity of singlet oxygen is generated at the initial state in any system. The rate constant increases with the unsaturated degree, except for oleic acid. As for oleic acid, its decomposition rate constant is comparably larger despite the lowest unsaturated degree.

Oxidizability of unsaturated fatty acids by active oxygen species in homogeneous bulk phases is accelerated with an increase in the number of their double bonds. In fact, the decomposition rate constants of oleic, linoleic, linolenic, and arachidonic acid methyl esters by singlet oxygen in pyridine are $0.7,1.3,1.9$, and $2.4 \times 10^{5} \mathrm{M}^{-1} \mathrm{Sec}^{-1}$, respectively ${ }^{17}$. Besides, the decomposition rate constants of linoleic, linolenic, and arachidonic acids against perhydroxyl radical in aqueous ethanoic solutions are 1.18, 1.70, and $3.05 \times 10^{3} \mathrm{M}^{-1} \mathrm{Sec}^{-1}$, respectively ${ }^{18}$. Therefore, the fast decomposition of oleic acid by singlet oxygen observed in this study is a unique phenomenon in the heterogeneous phospholipid bilayer membranes.

When solubilized by aqueous surfactant solutions, PpIX resides in the hydrocarbon region of surfactant micellar phases ${ }^{19)}$. We suggest from this viewpoint that PpIX is also incorporated in the hydrocarbon region of liposome bilayer

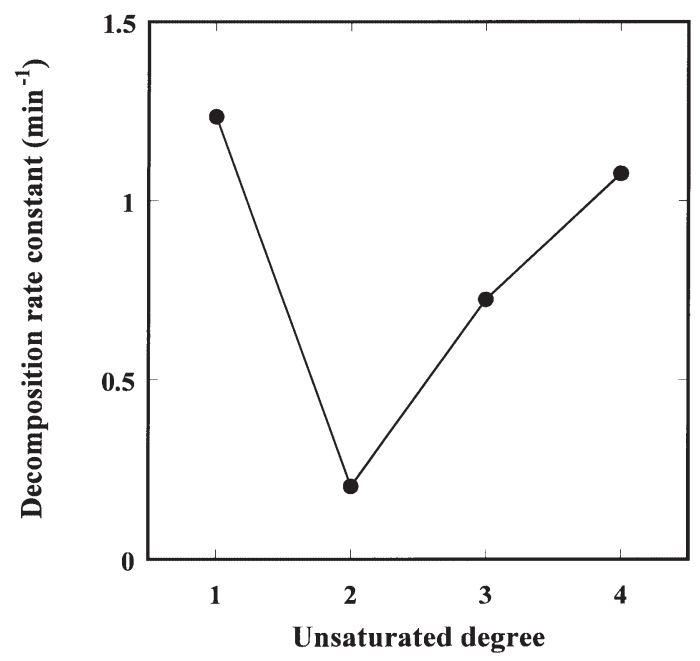

Fig. 6 Relationship between Decomposition Rate Constant at Initial State and Unsaturated Degree of Unsaturated Fatty Acid at $25^{\circ} \mathrm{C}$. membranes in the same manner. Moreover, unsaturated fatty acids are also penetrated into the hydrocarbon region of liposome bilayer membranes in parallel with the hydrocarbon chain of the phospholipid. Therefore, the oxidation decomposition of the unsaturated fatty acid by singlet oxygen generated from the photoexited PpIX is predicted to be closely related to properties of the hydrocarbon region of the liposome bilayer membrane.

In general, a lifetime of singlet oxygen is shortened with an increase in polarity (or permittivity) of its surroundings. For instance, lifetimes of singlet oxygen in water, acetone, and chloroform are $3.3,46.5$, and $60 \mu \mathrm{s}$, respectively ${ }^{11}$. Shorter lifetimes of singlet oxygen in polar solvents suppress its reactivity. When we measured $I_{1} / I_{3}$ of pyrene localized in the hydrocarbon region of the liposome bilayer membrane, shown in Fig. 7, micropolarity of the hydrocarbon region is almost constant in the case with any unsaturated fatty acid. Therefore, the micropolarity of the phospholipid bilayer membrane contributes little to the difference in the decomposition rate of the unsaturated fatty acid.

Increase in microfluidity of the hydrocarbon region in the phospholipid bilayer membrane accelerates mobility and diffusion of singlet oxygen and the unsaturated fatty acid in the membrane, promoting their oxidation reaction. According to the fast decomposition of oleic acid, it might be expected that microfluidity of the hydrocarbon region of the liposome bilayer membrane including oleic acid would be much greater. In measuring $\mathrm{I}_{\mathrm{E}} / \mathrm{I}_{\mathrm{M}}$ of pyrene in the hydrocarbon region in the liposome bilayer membrane, contrary to our expectation, the microfluidity is lower with oleic acid than with any other unsaturated fatty acid, as shown in Fig. 7. The fast decomposition of oleic acid cannot be then explained by the microfluidity of the hydrocar-

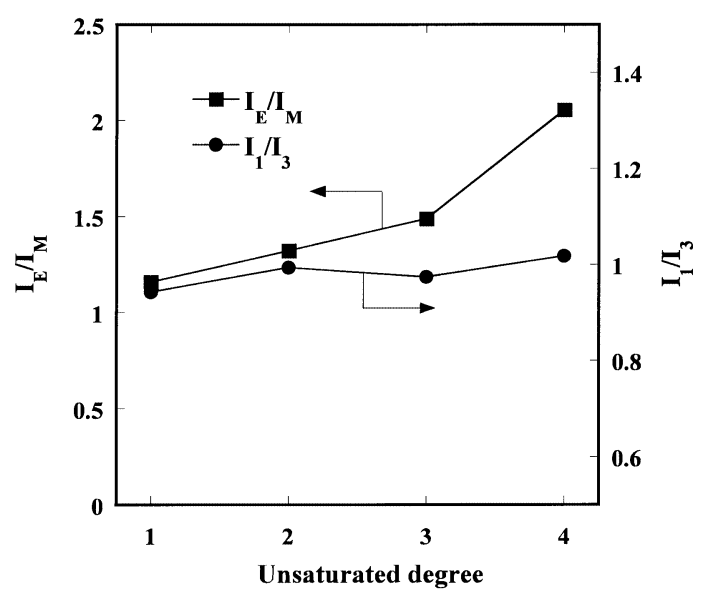

Fig. 7 Changes in $I_{E} / I_{M}$ and $I_{1} / I_{3}$ of Pyrene in Liposome Bilayer Membrane as a Function of Unsaturated Degree of Unsaturated Fatty Acid at $25^{\circ} \mathrm{C}$. 
bon region in the phospholipid bilayer membrane.

The intermolecular distance between the unsaturated fatty acid and PpIX in the liposome bilayer membrane is closely related to the decomposition rate of the unsaturated fatty acid. Unsaturated fatty acids with a cis-double bond have a pronounced bend, and this tendency is more remarkable for unsaturated fatty acids having higher unsaturated degrees. Hence, increasing unsaturated degree of unsaturated fatty acids spreads their molecule occupied areas, for example, $2.1 \mathrm{~nm}^{2}$ for stearic acid without a double bond, and 4.1 and $4.8 \mathrm{~nm}^{2}$ for oleic and linoleic

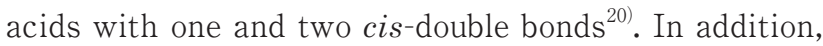
since $\mathrm{pK}_{\mathrm{a}}$ value of unsaturated fatty acids decreases with an increase in its unsaturated degree: 9.85 for oleic acid, 9.24 for linoleic acid, and 8.28 for $\alpha$-linolenic acid ${ }^{20)}$, the unsaturated fatty acid with lower unsaturated degrees tends to be protonated around neutral $\mathrm{pH}$. On the other hand, the $\mathrm{pK}_{\mathrm{a} 1}$ and $\mathrm{pK}_{\mathrm{a} 2}$ values of two carboxylic acids in PpIX evaluated from the $\mathrm{pK}_{\mathrm{a}}$ values of dicarboxylic metalloporphyrins are approximately 5 and 7 , respectively ${ }^{21}$, indicating that the carboxylic acids of PpIX seem to be deprotonated around neutral $\mathrm{pH}$. As a result, strong iondipole interaction acts between the carboxylate groups of PpIX and the carboxylic acid group of the unsaturated fatty acid with lower unsaturated degrees around neutral $\mathrm{pH}$. Tanking into consideration the smallest molecular occupied area and the strong ion-dipole interaction of the unsaturated fatty acid with lower unsaturated degrees, therefore, we suggest that the intermolecular distance between oleic acid and PpIX is shorter in the liposome bilayer membrane and that collision frequency between the cis-double bond of oleic acid and singlet oxygen generated from PpIX increases; thereby, resulting in the fast decomposition of oleic acid in the phospholipid bilayer membrane. However, this suggestion will need to be verified in future study.

\subsection{Characterization of liposome bilayer membrane after oxidation reaction}

In order to characterize the phospholipid bilayer membranes after the oxidation of the unsaturated fatty acid by singlet oxygen, we investigated irradiation time-dependence of the membrane micropolarity and microfluidity by measuring $I_{1} / I_{3}$ and $I_{E} / I_{M}$ of pyrene that was located in the hydrocarbon region of the membrane.

Changes in $\mathrm{I}_{1} / \mathrm{I}_{3}$ of pyrene in the liposome bilayer membrane with and without the unsaturated fatty acid are demonstrated as a function of light irradiation time in Fig. 8. Before this measurement, we determined no decay of the pyrene fluorescence under the light irradiation condition. As a result, the micropolarity in the hydrocarbon region of the membrane slightly decreases with increasing irradiation time in any system.

In addition, Fig. 9 shows changes in $\mathrm{I}_{\mathrm{E}} / \mathrm{I}_{\mathrm{M}}$ of pyrene with

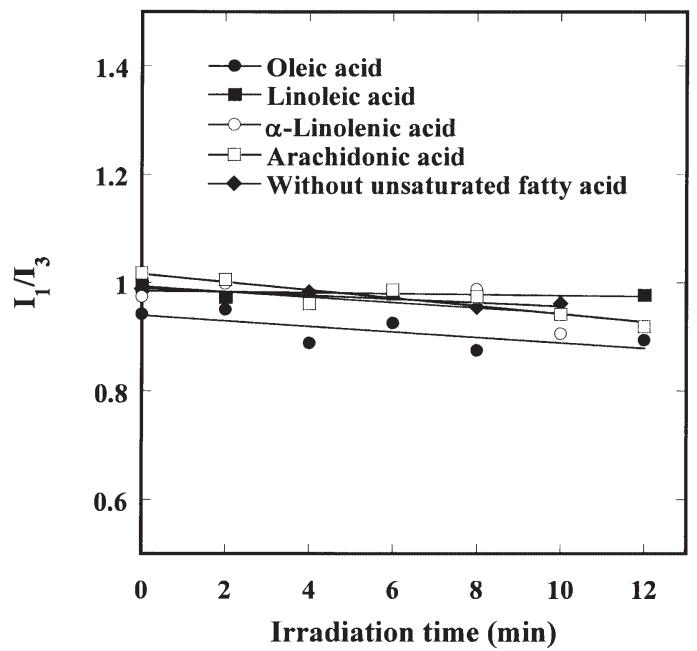

Fig. 8 Changes in $I_{1} / I_{3}$ of Pyrene in Liposome Bilayer Membrane as a Function of Light Irradiation Time at $25^{\circ} \mathrm{C}$.

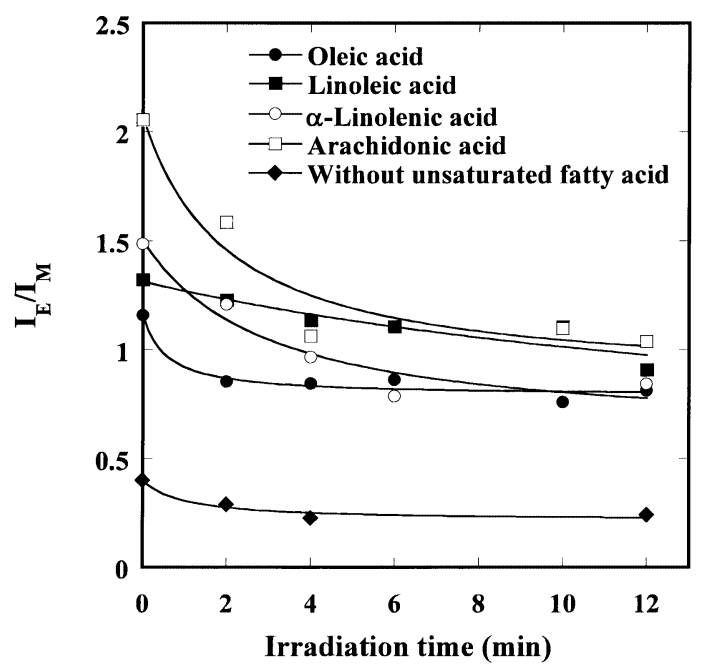

Fig. 9 Changes in $I_{E} / I_{M}$ of Pyrene in Liposome Bilayer Membrane as a Function of Light Irradiation Time at $25^{\circ} \mathrm{C}$.

light irradiation time. The microfluidity of the liposome bilayer membrane without the unsaturated fatty acid is almost constant, whereas the microfluidity with the unsaturated fatty acid is obviously reduced with an increase in irradiation time.

Singlet oxygen can easily react with a cis-double bond in unsaturated fatty acids through "ene" reaction, followed by formation of trans hydroperoxy group as follows ${ }^{22)}$. 


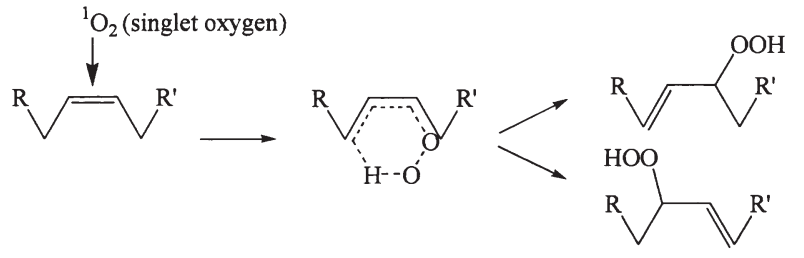

Hydroperoxy group introduces hydrophilicity to the unsaturated fatty acid. If the unsaturated fatty acid with hydroperoxy group remained in the hydrocarbon region of the liposome bilayer membrane, the micropolarity of the hydrocarbon region would rise as its oxidation reaction progresses. As seen from Fig. 8, nevertheless, the micropolarity is unexpectedly reduced with an increase in irradiation time. In addition, NMR measurement of the water bulk phase in the liposome dispersion after the light irradiation provided us no peak, as well as that before the light irradiation (the data is not shown). The unsaturated fatty acid with hydroperoxy group then seems to migrate from the hydrocarbon region into the hydrophilic region of the liposome bilayer membrane. Additionally, the migration of the oxidized unsaturated fatty acid makes the interaction among the hydrocarbon chains of DPPC promoted and induces close-packed and well-ordered orientation of the hydrocarbon chains, resulting in the reduction in the microfluidity of the hydrocarbon region, as shown in Fig. 9.

\section{CONCLUSION}

The unsaturated fatty acid was decomposed through the oxidation reaction with singlet oxygen generated by the photoexcited PpIX in the liposome bilayer membrane. The decomposition rate constant was obeyed as the following order: arachidonic acid $>$ oleic acid $>\alpha$-linolenic acid $>$ linoleic acid. Although having the smallest unsaturated degree, oleic acid was readily degraded. The short intermolecular distance between oleic acid and PpIX in the liposome bilayer membrane probably causes its fast decomposition. In addition, the micropolarity and microfluidity of the hydrocarbon region in the liposome bilayer membrane with the unsaturated fatty acid and PpIX decreased with an increase in light irradiation time. These results suggest that interaction among the hydrocarbon chains of DPPC in the liposome bilayer membrane is promoted by migration of the oxidized unsaturated fatty acid from the hydrocarbon region, leading to form close-packed and well-ordered orientation of the hydrocarbon chains.

\section{References}

1. Bonnett, R. Chemical aspects of photodynamic thera- py. Gorldon and Breach Science Publishers. Amsterdam. pp. 74-79 (2000).

2. Castano, A.P.; Demidova, T.N.; Hamblin, M.R. Mechanisms in photodynamic therapy: Part one-photosensitizers, photochemistry and cellular localization. Photodiagnosis Photodynamic Therapy. 1, 279-293 (2004).

3. Bonnett, R. Chemical aspects of photodynamic thera$p y$. Gorldon and Breach Science Publishers. Amsterdam. pp. 9-11 (2000).

4. Barnes, J.A.; Pehowich, D.J.; Allen, T.M. Characterization of the phospholipid and fatty acid composition of Sendai Virus. J. Lipid Research. 28, 130-137 (1987).

5. Lopez, R.F.V.; Lange, N.; Guy, R.; Bentley, M.V.L.B. Photodynamic therapy of skin cancer: Controlled drug delivery of 5-ALA and its esters. Advanced Drug Delivery Reviews. 56, 77-94 (2004).

6. Yazu, K.; Yamamoto, Y.; Ukegawa, K.; Niki, E. Mechanism of lower oxidization of eicosapentaenoate than linoleate in aqueous micelles. Lipids. 31, 337-339 (1996).

7. Yamamoto, Y. New developments in lipid peroxidation. Oleoscience. 1, 503-508 (2001).

8. Kobayashi, H.; Yoshida, M.; Maeda, I.; Miyashita, K. Proton NMR relaxation times of polyunsaturated fatty acids in chloroform solutions and aqueous micelles. $J$. Oleo Sci. 53, 105-108 (2004).

9. Bangham, A.D.; Standish, M.M.; Weissmann, G. The action of steroids and streptolysin $\mathrm{S}$ on the permeability of phospholipid structures to cations. J. Mol. Biol. 13, 253-259 (1965).

10. Lion, Y.; Delmelle, M.; Van de Vorst, A. New method of detecting singlet oxygen production. Nature. 263, 442443 (1976).

11. Saito, I.; Matsuura, T. Physicochemical aspects of molecular oxygen and active oxygen species. in Chemistry of Active Oxygen Species. (Chem. Soc. Jpn. eds.) Center for Academic Publications Jpn. Tokyo. pp. 6-9 (1990).

12. Thomas, J.K. Radiation-induced reactions in organized assemblies. Chem. Rev. 80, 283-299 (1980).

13. Benrraou, M.; Bales, B.L.; Zana, R. Effect of the nature of the counterion on the properties of anionic surfactants. 1. Cmc, ionization degree at the $\mathrm{cmc}$ and aggregation number of micelles of sodium, cesium, tetramethylammonium, tetraethylammonium, tetrapropylammonium, and tetrabutylammonium dodecyl sulfates. $J$. Phys. Chem. 107, 13432-13440 (2003).

14. Yokouchi, Y.; Tsunoda, T.; Inura, T.; Yamauchi, H.; Yokoyama, S.; Sakai, H.; Abe, M. Effect of adsorption of bovine serum albumin on liposomal membrane characteristics. Colloids Surf. B Biointerfaces. 20, 95-103 (2001).

15. Tokuoka, Y.; Niitsu, A.; Watabe, N.; Murakami, T.N.; Kawashima, N. ESR Spectroscopy of singlet oxygen generated by protoporphyrin IX in aqueous surfactant 
solutions. J. Oleo Sci. 52, 135-140 (2003).

16. Finlay, J.C.; Conover, D.L.; Hull, E.L.; Foster, T.H. Porphyrin bleaching and PDT-induced spectral changes are irradiance dependent in ALA-sensitized normal rat skin in vivo. Photochem. Photobiol. 73, 54-63 (2001).

17. Doleiden, F.H.; Fahrenholtz, S.R.; Lamola, A.A.; Trozzolo, A.M. Reactivity of cholesterol and some fatty acids toward singlet oxygen. Photochemistry and Photobiology. 20, 519-521 (1974).

18. Bielski, B.H.J.; Arudi, R.L.; Sutherland, M.W. A study of the reactivity of $\mathrm{HO}_{2} / \mathrm{O}_{2}^{-}$with unsaturated fatty acids. J. Biological Chem. 258, 4759-4761 (1983).

19. Maiti, N.C.; Mazumdar, S.; Periasamy, N. Dynamic of porphyrin molecules in micelles. picosecond timeresolved fluorescence anisotropy studies. J. Phys.
Chem. 99, 10708-10715 (1995).

20. Kanicky, J.R.; Shah, D.O. Effect of degree, type, and position of unsaturation on the $\mathrm{pK}_{\mathrm{a}}$ of long-chain fatty acids. J. Colloid Interf. Sci. 256, 201-207 (2002).

21. Kepczynski, M.; Ehrenberg, B. Interaction of dicarboxylic metalloporphyrins with liposomes. The effect of $\mathrm{pH}$ on membrane binding revisited. Photochem. Photobiol. 76, 486-92 (2002).

22. Adam, W.; Brunker, H.G.; Kumar, A.S.; Peters, E.M.; Peters, K.; Schneider, U.; Von Schnering, H.G. Diastereoselective singlet oxygen ene reaction (schenck reaction) and diastereoselective epoxidations of heteroatom-substituted acyclic chiral olefines: A mechanistic comparison. J. Am. Chem. Soc. 118, 18991905 (1996). 